\title{
A Biocompatible Dual-AIEgen System without Spectral Overlap for Quantitation of Microbial Viability and Monitoring of Biofilm Formation
}

\author{
Wei He ${ }^{\text {ac\# }}$ Zheng Zheng ${ }^{\text {a\#}}$, Haotian Bai ${ }^{\text {a\# }}$, Linghong Xiong ${ }^{\mathrm{b}}$, Lei Wang ${ }^{\mathrm{b}}$, Yinghui $\mathrm{Li}^{\mathrm{b}}$, \\ Ryan T. K. Kwok ${ }^{\mathrm{ac}}$, Jacky W. Y. Lam ${ }^{\mathrm{ac}}$, Qinghua Hü ${ }^{\mathrm{b}}$, Jinquan Cheng ${ }^{\mathrm{b} *}$, Ben Zhong \\ Tang ${ }^{\text {acd } *}$
}

${ }^{a}$ Department of Chemistry, Department of Chemical and Biological Engineering, Hong Kong Branch of Chinese National Engineering Research Center for Tissue Restoration and Reconstruction, Institute of Advanced Study and Division of Life Science The Hong Kong University of Science and Technology, Clear Water Bay, Kowloon, Hong Kong, China

${ }^{\mathrm{b}}$ Shenzhen Center for Disease Control and Prevention, Shenzhen 518055, China

${ }^{c}$ HKUST-Shenzhen Research Institute, No. 9 Yuexing 1st RD, South Area, Hi-tech Park Nanshan, Shenzhen 518057, China

${ }^{\mathrm{d}}$ Center for Aggregation-Induced Emission, SCUT-HKUST Joint Research Laboratory and State Key Laboratory of Luminescent Materials and Devices, South China University of Technology, Guangzhou 510640, China

\begin{abstract}
Lacking rapid and reliable microbial detection and sensing platforms, insufficient understanding towards microbial behaviors may generated or delayed precautions could be made, which greatly threatens human life and increase heavy financial burdens to the society. In this contribution, a dual-aggregation-induced emission luminogen (AIEgen) system is successfully developed for microbial imaging and metabolic status sensing. This system consists of two AIEgens (DCQA and TPE-2BA) that bear positive charged groups or boric acid groups, providing universal microbial staining ability and specific affinity to dead microbes, respectively. On the basis of the distinctive fluorescence response produced by the diverse interaction of AIEgens with live or dead microbes, this dual-AIEgen system can detect all the microbe and identify their viabilities. Furthermore, the morphology and metabolic status of a sessile biofilm can also be imaged and monitored. The system exhibits rapid labelling, suitable for various microbes, and good biocompatibilities.
\end{abstract}




\section{Introduction}

Microorganisms are the most aged and most abundant population on earth. These tiny organisms are all over the place but yet we could not see them by naked eyes. In total, there are around 1,400 known species of pathogenic microbes (including viruses, bacteria and fungi), but it only accounts for much less than $1 \%$ of the total number of microbial species on the planet. ${ }^{1-3}$ The history of mankind is a continuous battle against these invisible microbes. ${ }^{4-8}$ For example, the Black Death, one of the deadliest pandemic recorded in human history, resulted in up to 75-200 million deaths throughout recorded history. The bacterium Yersinia pestis was identified as the culprit of the plague pandemic in 1894, after which transmission of bacteria from rodents by flea bites was discovered in 1898. The plague was finally controlled until the use of antibiotics started from 1930s. ${ }^{9}$ Indeed, the plague became an impetus for significant developments in medicine and imaging tools for microbial detection and identification.

Several methods have been commonly used for microbial detection in clinic and research such as Gram staining, plate counting, minimum inhibition concentration (MIC) test and electron microscopes (EM). ${ }^{10-13}$ Among them, Gram staining is the most widely used method to distinguish Gram-positive from Gram-negative bacteria on the basis of differential staining with a crystal violet-iodine complex and a safranin counterstain. The cell walls of Gram-positive organisms retain this complex after treatment with alcohol and appear purple, whereas gram-negative organisms decolorize following such treatment and appear pink. ${ }^{14}$ But it requires washing and counterstaining steps, and it can only roughly classify bacteria into two giant categories regardless of live/dead status. Plate counting method is developed to quantify the live bacteria by spread diluted bacteria suspension on proper agars. While it takes at least 24-hour incubation time, and only a narrow diversity of microbes can be cultured accurately on the appropriated medium agar. ${ }^{10,15}$ On the other hand, MIC is the most commonly adopted method to evaluate the antimicrobial ability of drugs and biomaterials. However, the readout of the MIC array is depended on the turbidity by visual inspection which is not sensitive enough and may generate system errors in practice. ${ }^{16}$ The application of SEM and TEM enable researchers to observe the fine structures of microbes, though these techniques require sophisticated sample preparation. Unfortunately, the four techniques mentioned above are not capable to characterize the microbes in-situ, which are hard to meet the requirement of real-time monitoring of 
dynamic microorganism. Unrevealing of microbial dynamic biological processes could provide us more information and strategies to fight against microbes. Therefore, it is promising to further promote technological innovation and realize the visualization of microbes in-situ. ${ }^{17-20}$

Fluorescent imaging technology has a lot of advantages, such as high sensitivity, nanosize resolution and in-situ/on-site visualization capabilities. ${ }^{20-23}$ Many fluorescent probes have been developed for microbial investigations, but they suffer from the intrinsic defect of aggregation caused quenching (ACQ) effect. So far, most of the commercial fluorescent probes are in hydrophobic nature, so they trend to form aggregates in aqueous phase. ${ }^{24}$ The resulting multiple intermolecular $\pi-\pi$ interactions lead fluorescence decrease or even complete quenching. Additionally, this drawback is further exacerbated in complex biological system, and prevents these traditional fluorescent probes from being incorporated into the biofilms. Microbial biofilm is the most common life form in nature for microbes, fast adaption to their environment, drug resistance generation and cooperation with others could be achieved in biofilm state. Biofilm is that aggregates of microorganisms in which cells are frequently embedded in a self-produced matrix of extracellular polymeric substances (EPS) that are adherent to each other and/or a surface. ${ }^{25-29}$ In microorganism world, the aggregated style is more common but complicated, and lack of enough understanding. As a biofilm contains complicated components, such as large numbers of microbes and water-rich matrixs, ${ }^{28,}$ ${ }^{29}$ while the ACQ probes tends to form aggregates so that cannot work efficiently at such a microenvironment. ${ }^{24}$ To avoid the ACQ effect, the use of fluorescent probes at low concentration is the only choice, although it results in incomplete information of the biofilm. Besides, the traditional fluorescent probes such as propidium iodide (PI) and SYTO 9 (popular in commercial bacterial imaging kit) always suffer from their toxicity. To reduce the interference of high toxicity, these probes have to be washed away from the samples before fluorescent imaging. ${ }^{30}$ Whereas any additional washing procedures seem to be alter the morphology and structure of biofilms. ${ }^{31,32}$ In considering of spectral overlap, the SYTO 9 and PI pair would introduce inevitable system errors into results. Therefore, it would be fascinating if a biocompatible system for fluorescent microbial imaging with wash free and minimum spectra overlap properties is developed. 
Neoteric fluorescent materials with aggregation-induced emission characteristic can meet all the above requirements. AIE luminogens (AIEgens) are non-emissive when molecularly dissolved in solvents, but exhibit intense fluorescence in the aggregation state. ${ }^{24,} 33,{ }^{34}$ AIEgens have lots of advantages such as tunable chemical structure, adjustable emission spectrum, good biocompatibility, and high potential to be used as "wash-free" and "light-up" probes. ${ }^{35}$ Plenty of AIEgens have been employed for bacteria imaging and sensing. ${ }^{36-38}$ However, it is very important to image the biofilm morphology and the viabilities of biofilm cells at the same time. A dual-AIEgen system is a promising solution for this problem and fulfills the demands. Specifically, in such a system, two components are incorporated by rationally tuned their targeting abilities and photophysical properties. ${ }^{39,40}$ For the first component, a NIR AIEgen probe can be easily designed to possess a broad spectrum targeting ability, that endows this component the ability to stain all the microbes with a high efficiency. Then by tuning the donor- $\pi$-acceptor structure, red emissive AIEgens can be obtained. ${ }^{41,}{ }^{42}$ AIEgens with long wavelength excitation can minimize the autofluorescence from microbes, so it can enhance the imaging resolutions. Besides, deep penetration depth can be achieved when using red emissive AIE probes. ${ }^{43}$ Next, two positive charges have been introduced into the structure with a short carbon chain, that can be conducted to achieve universal targeting ability and good biocompatibility at the same time. ${ }^{44,45}$ In order to realize the minimum spectral overlap, the second component should be blue enough. However, UV is harmful to biological samples, thus a blue AIEgen (TPE-2BA) is selected. TPE2BA is a cell-impermeable DNA stain that binds to the groove of double-stranded DNA. Bacteria with compromised membrane open the access for TPE-2BA to reach DNA, endowing it with strong emission. ${ }^{46}$

In this work, a dual-AIEgen system composed of a new NIR-emissive AIEgen (DCQA) and a blue-emissive AIEgen (TPE-2BA) with minimum spectral overlap has been designed and fabricated for microbial viability quantification and biofilm formation monitoring. The newly synthesized DCQA has been demonstrated to possess the wide spectrum staining ability towards all kinds of microbes, including gram-positive bacteria, gram-negative bacteria and fungi. The metabolic status of planktonic microbes and their biofilms could be successfully monitored by DCQA in combination with TPE2BA, which is a blue fluorescent probe for specific dead microbe imaging. Moreover, the quantification ability of this dual-AIEgen system has been verified by confocal 
microscope imaging, plate reader and flow cytometry. Finally, the dual-AIEgen syetem has been successfully applied for three-dimensional (3D) imaging of three kinds microbial biofilms.

\section{Results and discussion}

A fluorophore decorated a strong donor-acceptor (D-A) pair could facilitate intramolecular charge transfer (ICT) resulting in small electronic bandgaps and long absorption and emission wavelengths. ${ }^{35,47}$ Besides the electron-withdrawing potency, the introduction of electron acceptors with positive charges could potentially endow fluorophores with unique biological functions of universal microbial targeting and imaging abilities through electrostatic interaction with negative charged microbial membranes. ${ }^{13}$ Based on this design strategy, a NIR-emissive AIEgen, DCQA comprising a quinolinium salt as electron acceptor, a carbazole fragment as $\pi$ bridge and a diphenylamine as electron donor was synthesized by typical Knoevenagel condensation in a high yield and its chemical structure was fully characterized by NMR and mass spectrometry (Fig. 1A, Scheme S1, Fig. S1\&2). Prior to the optical studies, density functional theory (DFT) calculations of this new molecule were performed to understand their ICT. As shown in Fig. S3, the electron clouds of HOMOs of DCQA were mainly located on the diphenylamino moiety and carbazole framework, while the LUMOs were primarily contributed by the orbitals of the quinolinium moiety and the carbazole ring.

The photophysical properties of DCQA were subsequently investigated by UV-vis and photoluminescence (PL) spectroscopies. As depicted in Fig. S4A, DCQA showed an absorption maximum at $519 \mathrm{~nm}$ in dimethylsulfoxide (DMSO) attributed to ICT from the diphenylamino moiety to the quinolinium group. The AIE properties of DCQA was studied in DMSO/toluene mixtures with different toluene fractions (Fig. 1B and 1C). This compound showed very weak emission in DMSO due to the active intramolecular motions, which consumed the energy of the excitons through nonradiative pathways. Upon gradual addition of toluene to the DMSO solution, the fluorescence of the solution became stronger. At 99 vol \% toluene fraction, the fluorescence intensity of DCQA at $729 \mathrm{~nm}$ was 306-fold higher than that in the pure DMSO solution. These results demonstrated that DCQA was AIE active and emitted NIR light peaked at 729 $\mathrm{nm}$. Owing to the AIE characteristics, DCQA showed bright solid-state fluorescence 
with quantum yield of $1.2 \%$ determined by an integrating sphere (Fig. 1C). Timeresolved fluorescence measurement of DCQA in the solid state revealed lifetime of 1.484 ns. (Fig. S4B). Moreover, DCQA showed very large Stokes shift ( $\Delta v=210 \mathrm{~nm}$ ), which is favorable for bio-imaging due to the little signal overlap between photoexcitation and emission.
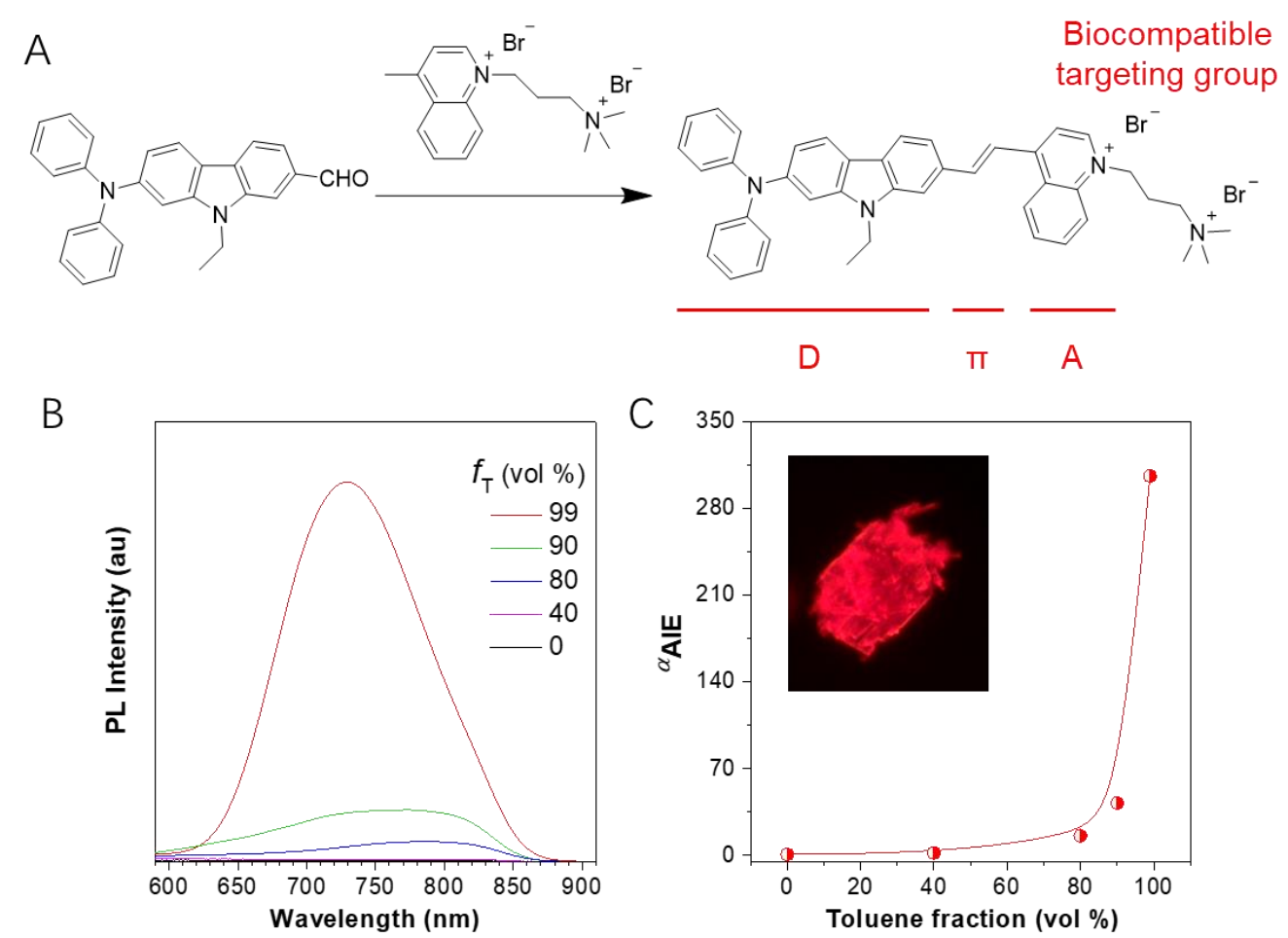

Figure 1. (A) Design rationale and synthetic route to NIR AIEgen DCQA. (B) Photoluminescence (PL) spectra of DCQA in DMSO/toluene mixtures with different toluene fraction (vol\%). (C) Plot of $\alpha_{\mathrm{AIE}}\left(I / I_{0}\right)$ versus the composition of the DMSO/toluene mixture of DCQA, where $I_{0}$ was the PL intensity at ?? nm at $f_{\mathrm{T}}=0 \mathrm{vol} \%$; concentration $=10 \mu \mathrm{M} ; \lambda_{\mathrm{ex}}=519 \mathrm{~nm}$. Inset: fluorescent microscope image of the DCQA powder. $\lambda_{\mathrm{ex}}=510-550 \mathrm{~nm}$. $\lambda_{\mathrm{em}}=590-800 \mathrm{~nm}$ ).

The integration of AIE characteristics, large Stokes shift, NIR emission and two positive charges with a short carbon chain makes DCQA a promising candidate for biological applications. Microbial imaging experiments were initially conducted by incubating DCQA with three indicator type strains, which were S.aureus (representation of Gram-positive bacteria), E. coli (representation of Gram-negative bacteria), and C. albicans (representation of fungi) respectively, followed by CLSM imaging excited at $560 \mathrm{~nm}$ (Fig. 2A-C). All three kinds of microbes either in live or dead forms were fluorescently stained by DCQA with very high efficiency. The 
incubation time test indicated that DCQA could stain all kinds of microbes in a short time ( $<5 \mathrm{~min}$ ) with strong fluorescence (Fig. S5). After that, the DCQA fluorescence signal reached the maximum, indicating that DCQA had a proper staining window for both imaging and high throughput analysis. This result demonstrated that DCQA could be applied as a universal detection probe for highly efficient and rapid staining of all kinds of microorganisms, Fig. 2D-E show the on-site fluorescence spectra of DCQA recorded by confocal microscope in different microbes. For all S. aureus, E. coli and $C$. albicans, NIR emission of DCQA was detected in both live and dead ones. In order to investigate the targetability of DCQA, negative-charged bacterial components including lipopolysaccharide (LPS), DNA and RNA were chosen to interact with DCQA (Fig. S6). The PL results indicated that DCQA emitted strong fluorescence when interacting with these three negative charged components because of electrostatic interactions. This is consistent with the phenomenon observed in fluorescence imaging, the whole microbial cell was lighted up by DCQA.
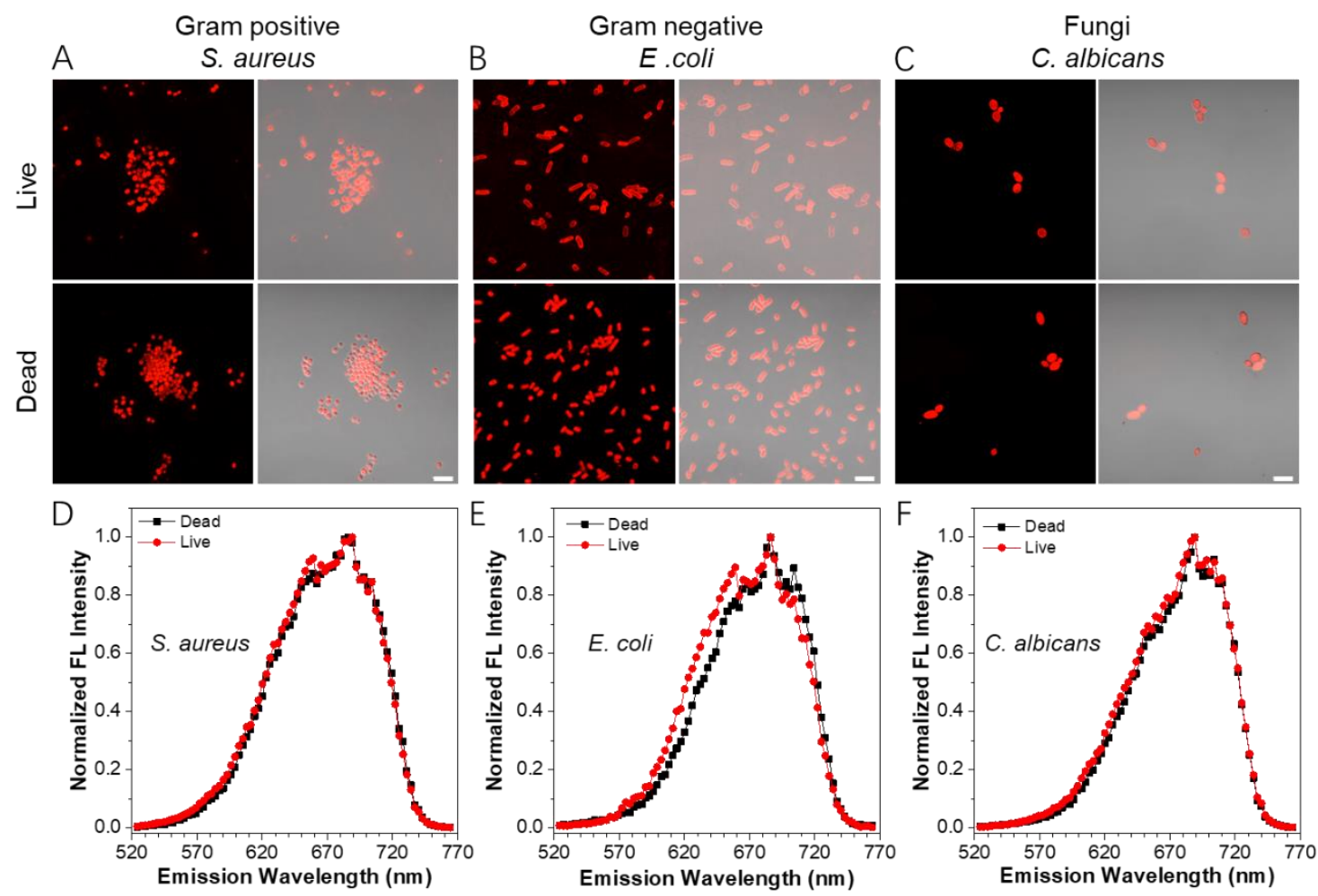

Figure 2. Staining microbes by AIE-active DCQA. (A-C) CLSM images of living microbes (upper panel) and dead microbes (lower panel) stained with DCQA. Excitation wavelength: $561 \mathrm{~nm}$. Emission range: 590-759 nm. Scale bars: (A) S. aureus; $=5 \mu \mathrm{m}$; (B) E. coli $=5 \mu \mathrm{m}$; (C) C. albicans $=10 \mu \mathrm{m}$. (D-F) in-situ emission spectra of 
DCQA interacted with (D) S. aureus, (E) E. coli and (F) C. albicans recorded by CLSM. Excitation wavelength: $561 \mathrm{~nm}$. Emission range: 530-780 nm. Concentration of DCQA was $10 \mu \mathrm{M}$.

Microbial metabolism status is closely related to their functionality, biofilm formation ability, virulence factor secretion, drug resistance generation and other characteristics. ${ }^{48}$ While it could be changed in different environment. Especially for opportunistic pathogens, the change of their metabolic level might lead to harmful situation for human beings. ${ }^{48,49}$ The viability of microbes is the key factor of microbial metabolism. It has been reported that part of bacteria sacrifice themselves to disperse their drug resistance genes to the rest when they are confront of specific antibiotics. ${ }^{28}$ The rest of the microbe species receive the drug resistance genes and acquire resistance to specific antibiotics. Therefore, it is very important to monitor the metabolic status of microbes. In this work, the factor of microbial viability was chosen as a standard. ${ }^{50}$ In order to achieve minimum emission spectral overlap with DCQA, the metabolic sensor probe should have a proper emission spectrum to match with that of DCQA. Previously reported TPE-2BA was selected as the synergetic metabolic sensor. This combination of DCQA and TPE-2BA with negligible spectra overlap could make them a perfect dual-AIEgen system for microbial viability monitoring and morphological tracking.

To evaluate the ability of this dual-AIEgen system for monitoring microbial metabolic status, three categories of microbes including Gram-positive, Gram-negative and fungi were applied in the imaging experiments. Moreover, in each category, two most frequently studied type strains were selected. S. aureus and B. sublitis represent Grampositive bacteria. E. coli and $S$. marcescens represent Gram-negative bacteria. $C$. albicans and $S$. cerevisiae represent fungi. Each microbe that was in the period of logarithmic growth was divided into two groups. One group was treated with $75 \%$ ethanol, which can effectively kill the microbes. Then washed with $1 \times$ PBS. Another group was washed with $1 \times$ PBS only (Fig. S7). Then the two groups were mixed at a ratio 1:1 to prepare the samples. After $30 \mathrm{~min}$ incubation with the dual-AIEgen system, the confocal images of microbes were acquired. In Fig. 3A, DCQA channel (590 - 759 $\mathrm{nm}$ ) showed that all the Gram-positive bacteria were stained and imaged. TPE-2BA channel (410-560 nm) indicated the dead ones effectively. Live and dead bacteria could be easily readout by merging two channels, where red represent live and blue represent dead bacteria. For Gram-negative bacteria and fungi, same results could be obtained by 
confocal microscope as shown in Fig. 3B and 3C. In this system, DCQA was responsive for overall microbial cells in order to detect the accurate amount of total microbes, while TPE-2BA was responsive for microbial viability detection. The viability of Gram-positive bacteria, Gram-negative bacteria and fungi could be calculated accurately through the precise determination of the amount of dead and total microbial cells.

The influence of the dual-AIEgen system toward bacterial viability was further investigated by MIC assay (Table S1). The MIC values of DCQA and TPE-2BA towards three indicating strains, S. aureus, E. coli and C. albicans were over $80 \mu \mathrm{M}$ and $400 \mu \mathrm{M}$, respectively. Both of which were eight times higher than their working concentration. The results indicated that the two components showed no dark toxicity to the microbes, and this dual-AIEgen system was suitable for microbial morphology and viability detection and visualization.

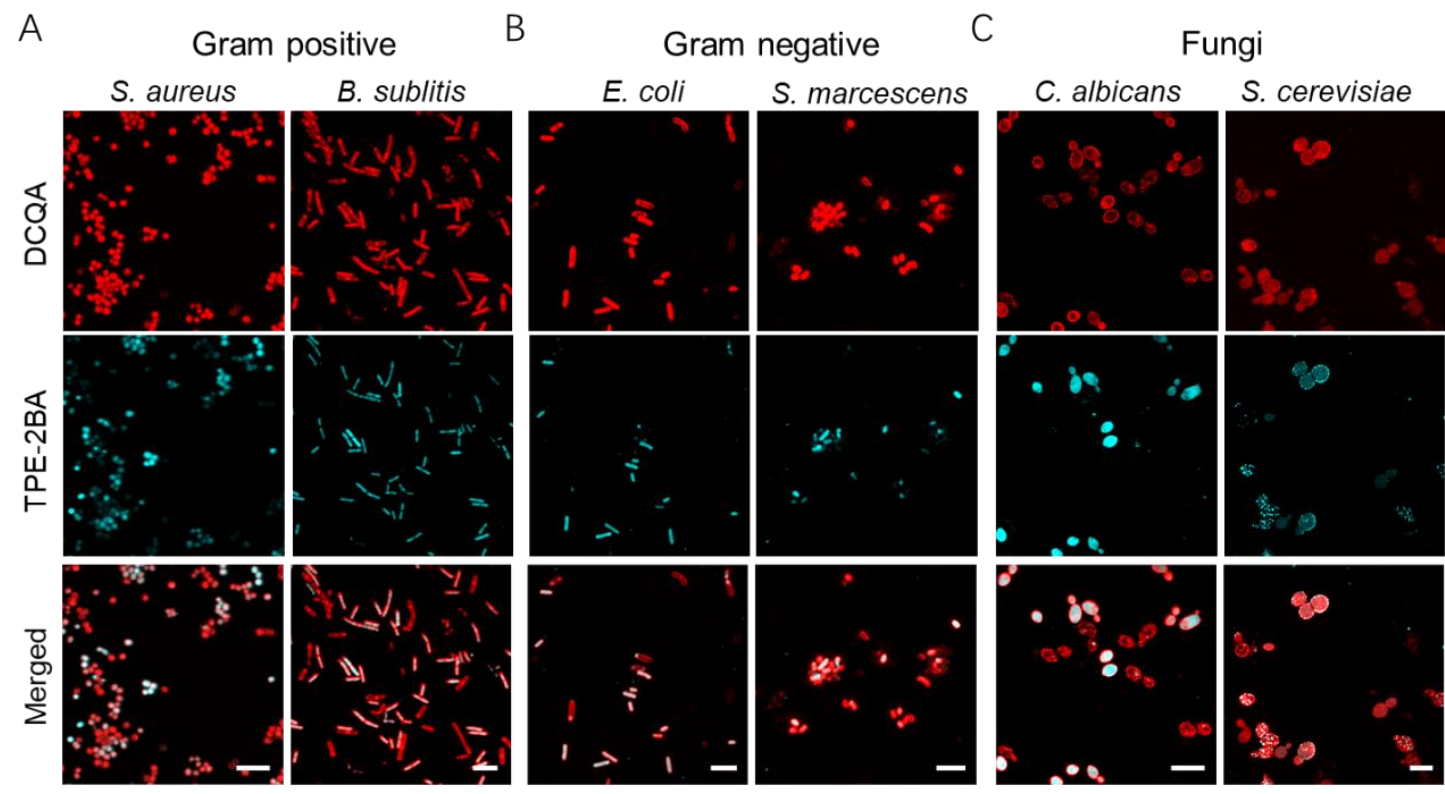

Figure 3. CLSM images of living and dead mixture of microbial cells co-stained with DCQA and TPE-2BA. (A) Gram positive bacteria. Left: S. aureus. Right: B. sublitis. Scale bar $=5 \mu \mathrm{m}$. (B) Gram negative bacteria. Left: E. coli. Right: S. marcescens. Scale bar $=5 \mu \mathrm{m}$. (C) Fungi. Left: C. albicans. Right: S. cerevisiae. Scale bar $=10 \mu \mathrm{m}$. Excitation wavelength: $561 \mathrm{~nm}$ for DCQA and $405 \mathrm{~nm}$ for TPE-2BA. Emission ranges: $590-759 \mathrm{~nm}$ for DCQA and 410-560 nm for TPE-2BA. Concentrations of DCQA and TPE-2BA are $10 \mu \mathrm{M}$ and $50 \mu \mathrm{M}$, respectively. 
Except for the above qualitative analysis, the more meaningful quantitative analysis was well processed by our dual-AIE system. During the development of antibiotics and antimicrobial materials, characterizing and quantifying the killing efficiency of these materials were important and required. First, plate reader was adopted due to its high throughput and large sample capacity properties. Live and dead E. coli were mixed together at different ratios to make the given percent live bacteria. Then the bacteria were co-stained by DCQA and TPE-2BA for $30 \mathrm{~min}$. Finally, plate reader was used to measure the peak intensities under different excitation wavelength. The results were shown in Fig. 4A, the red/blue fluorescence ratio had a positive linear correlation to the given percent live bacteria mixtures from 0 to $100 \%$, indicating the dual-AIEgen system was able to characterize the microbial viability by using plate reader efficiently and reliably.

Furthermore, due to the minimal spectral overlap between DCQA (730 nm) and TPE2BA (460 nm), crosstalk had been avoided and system errors reached the least. Then, confocal microscope was conducted to investigate the microbial viability distinguishing ability of this dual-AIEgen system. The sample preparation process was same as that in plate reader method. After staining for $30 \mathrm{~min}$, the bacterial mixtures with different live bacteria percentage were imaged by confocal microscope. More than 300 bacterial cells were collected for each mixture. As shown in Fig. 3, the total number of bacterial cells can be obtained through DCQA channel $\left(\mathrm{N}_{\text {total }}\right)$ and the number of dead ones can be calculated by using TPE-2BA channel $\left(\mathrm{N}_{\text {dead }}\right)$. Then the counted percentage of live bacteria $(L \%)$ could be calculated by Equation 1.

\section{Equation 1:}

$$
L \%=\frac{\mathrm{N}_{\text {total }}-\mathrm{N}_{\text {dead }}}{\mathrm{N}_{\text {dead }}}
$$

The setup of confocal microscope for DCQA was $560 \mathrm{~nm}$ as excitation, $600-750 \mathrm{~nm}$ as emission range. For TPE-2BA, $405 \mathrm{~nm}$ was chosen as excitation wavelength, 420-500 $\mathrm{nm}$ were chosen as emission range. There was no crosstalk between the two channels. It can be observed in Fig. 4B, the red/blue fluorescence ratio had a good linear correlation to the given percent live bacteria mixtures from 0 to $100 \%$, indicating the dual-AIEgen system was able to characterize the microbial viability by using confocal microscope efficiently and reliably. 
Flow cytometry had also been applied to test the staining ability of dual-AIEgen system. Flow cytometry is a powerful technique in biological research area which can provide high throughput analysis of different kinds of bio-samples, such as cells and microbes. By reading the fluorescence signals from labeled microbes, the microbial viability could be acquired and analyzed. After staining for $30 \mathrm{~min}$, mixtures of different live bacteria percentage were analyzed by flow cytometer, 5000 bacterial cells were analyzed for each mixture. In Fig. 4C and D, histogram and linear fitting results were presented.
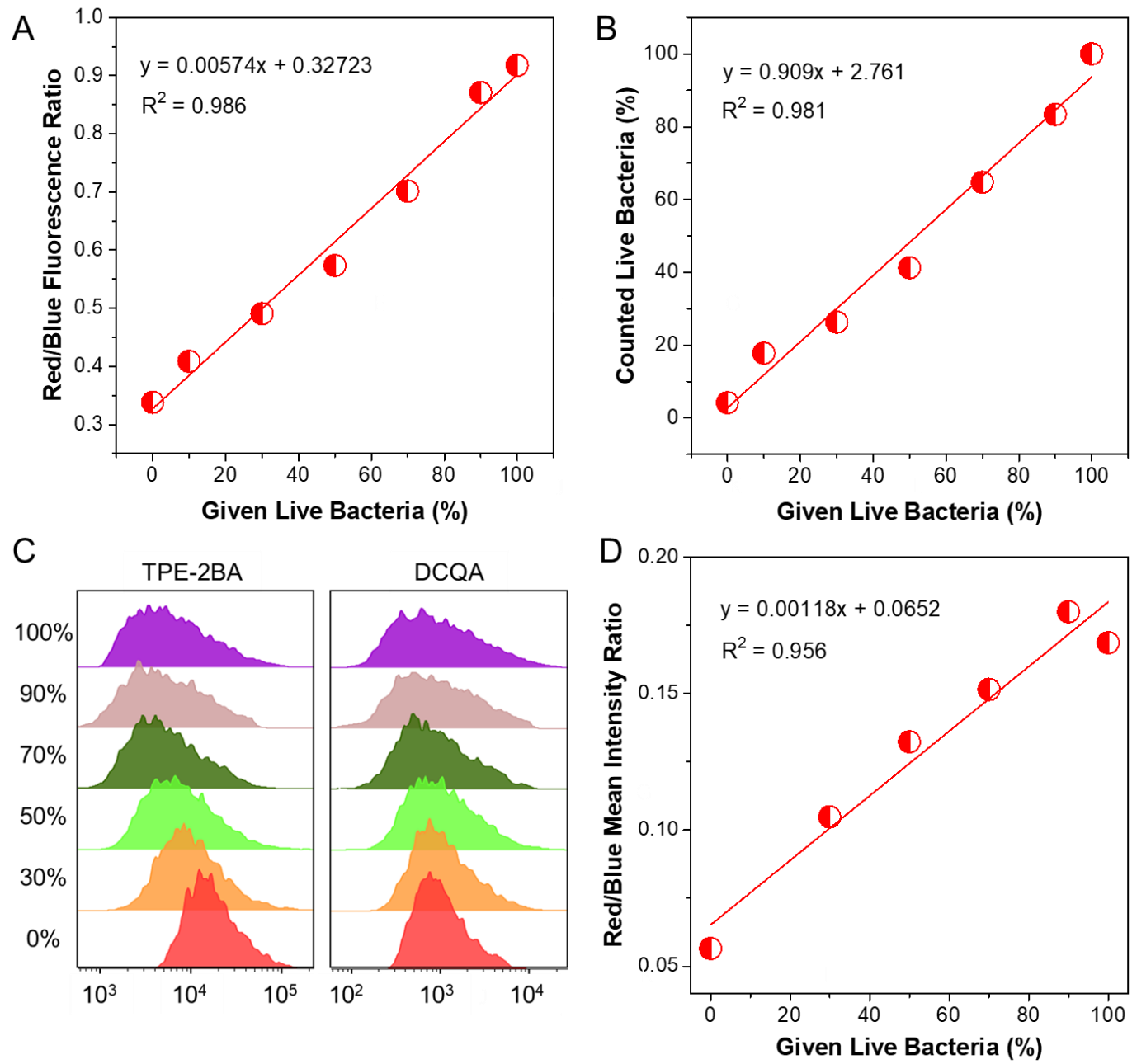

Figure 4. Quantification study of DCQA and TPE-2BA mixture. (A) Analysis of relative viability of $E$. coli suspensions by microplate reader. For blue channel: $\lambda_{\mathrm{ex}}=$ $310 \pm 10 \mathrm{~nm}, \lambda_{\mathrm{em}}=460 \pm 5 \mathrm{~nm}$. For red channel: $\lambda_{\mathrm{ex}}=519 \pm 10 \mathrm{~nm}, \lambda_{\mathrm{em}}=730 \pm 5 \mathrm{~nm}$. Each point represents the mean of four measurements. (B) Analysis of relative viability of E. coli suspensions by confocal microscope. (C) Statistical analysis of E. coli suspensions by flow cytometry. For TPE-2BA channel: $\lambda_{\mathrm{ex}}=405 \mathrm{~nm}, \lambda_{\mathrm{em}}=450 \pm 40$ 
$\mathrm{nm}$. For DCQA channel: $\lambda_{\mathrm{ex}}=561 \mathrm{~nm}, \lambda_{\mathrm{em}}=780 \pm 60 \mathrm{~nm}$. (D) Analysis of relative viability of $E$. coli suspensions by mean intensity obtained by flow cytometry. The mean intensities of the blue $(450 \pm 40 \mathrm{~nm})$ and red $(780 \pm 60 \mathrm{~nm})$ emission of suspensions were used for calculation.

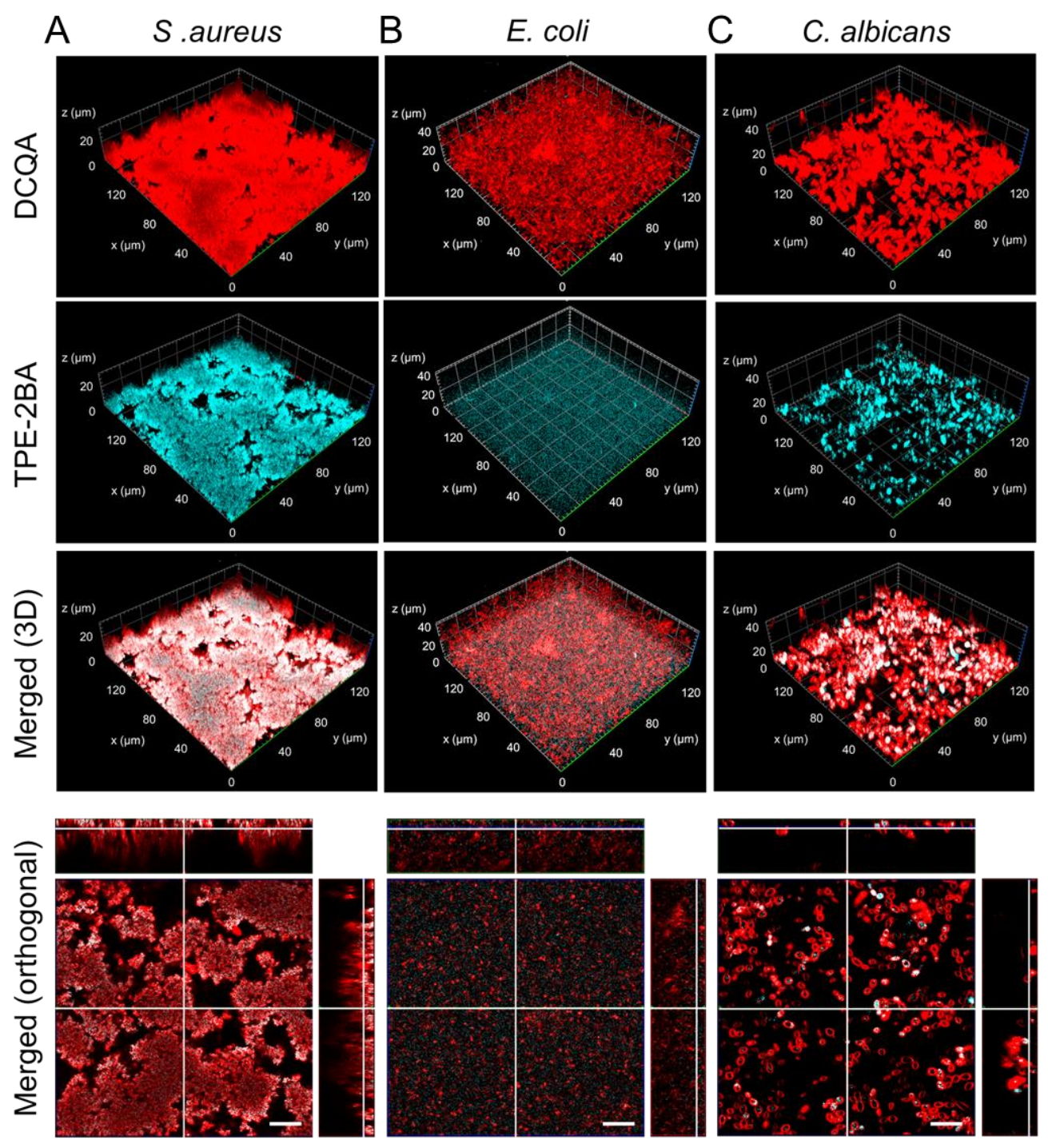

Figure 5. Biofilm morphology and metabolic status monitoring realized by combination of DCQA and TPE-2BA. CLSM images of microbial biofilms (three days old) stained by DCQA and TPE-2BA. (A) S. aureus. (B) E. coli. (C) C. albicans. Scale bar: $20 \mu \mathrm{m}$.

Bacterial biofilm is a form of bacteria aggregate embeded in a complicated excellular matrix. Biofilm endows bacteria with different behaviors and functions, such as antibiotic-resistence, strong connections and collaborations. ${ }^{51}$ Therefore, there is an 
urgent demand for visualization of its structures and functions. As demonstrated above, the dual-AIEgen system was effective for planktonic microbe imaging and viability identification, indicating this system has great potential for biofilm imaging. Thus three kinds of fundamental microbial biofilms, i.e. S. aureus biofilm, E. coli biofilm and $C$. albicans biofilm, were selected for the demonstration (Fig. 5). From the first panel of Fig. 5A-C, it can be found that the morphologies had been depicted by DCQA which is believed to stain all the microbial cells in the biofilm. As can be found in S. aureus biofilm, the mushroom-like morphology had been well stained and presented by DCQA. While loose morphology is the characteristic of E. coli biofilm which had been well stained and presented by DCQA too (Fig. 5B). The thickness was found to be around $40 \mathrm{um}$, more thick than $S$. aureus biofilm. This is because E. coli bacterial cells were dispersed much more loosely than those in $S$. aureus biofilm. The difference of two dominating bacterial biofilm models had been illustrated by DCQA successfully. $C$. albicans biofilm with typical morphology was also successfully imaged by DCQA (Fig. 5C). These results were consistent with the design principle that red emissive DCQA was responsible for monitoring the morphology based on its broad staining property and low toxicity. Two positive charges endowed DCQA universal staining ability towards negative charged microbes through electrostatic interaction efficiently and firmly. In the second panel of Fig. 5A-C, metabolic status of each biofilm had been achieved and visualized by blue emissive AIEgen TPE-2BA. During the process of a biofilm growing, there are four stages, bacterial adhesion, biofilm formation, biofilm maturation and biofilm dispersal. In a mature biofilm, part of the microbial cells are dead due to the natural life cycle and development of biofilm structure and functions. When the biofilm reached mature stage, the core area tended to be hypoxia and less nutritious, thus there were more dead cells inside. ${ }^{26,28,29}$ This phenomenon was significantly shown in mushroom-like $S$. aureus biofilm (second panel, Fig. 5A) ${ }^{52}$ Dead S. aureus cells located at the bottom and core area of the biofilms, which was consistent with discriptions in classical biofilm models. While for E. coli biofilm in Fig. 5B, it looked like a water-absorbing foam where bacterial cells were dispersed randomly which was due to the polysaccharide-rich extracellular matrix. ${ }^{53}$ Because of loose packing, E. coli biofilm was a less hypoxia environment so that less dead cells were found there. For $C$. albicans biofilm, most dead cells were found to be located at aggregated sites. This was attributed to the natural life cycle of fungi cells. ${ }^{27}$ In the third panel of Fig. 5A-C, merged images of DCQA and TPE-2BA were presented. It is more 
straightforward to observe the morphology and viability of the biofilms at the same time. The morphology could be observed directly in 3D merged images, at the same time the viability could be reported by color where blue or white represent dead ones and red represent live ones. Meanwhile, the distribution of the dead cells could be indicated more obviously along with the total biofilm morphology as background. The orthogonal views further demonstrated that biofilm morphology and viability could be visualized by the dual-AIEgen system.

In conclusion, a NIR emissive AIEgen, DCQA, was successfully designed and synthesized. The precision molecular design endowed the DCQA with unique AIE properties and good biocompatibility. The broad spectrum staining ability of DCQA can be successfully applied for in-situ and real-time imaging of gram-positive bacteria, gram-negative bacteria and fungi in both live and dead status. In combination with TPE2BA that only stained dead microbes, a dual-AIEgen system with minimal spectral overlap for quantitation of microbial viability and monitoring of biofilm formation was successfully constructed. Three categories of microbes (Gram-positive bactera, Gramnegative bacteria and fungi) were employed to demonstrate that the dual-AIEgen system could be applied in a wide range of microbes. In addition, different highthroughput evaluations were carried out to investigate the dual-AIEgen system and results confirmed its quantitation ability for the live-dead bacterial cell ratio. Moreover, biofilm imaging results indicated that this system was suitable for the visualization and monitoring of sessile microbes' morphology and metabolic status. This dual-AIEgen system could be applied for microbial imaging and it is a promising kit for the development of antimicrobial biomaterials.

\section{Methods}

\section{Materials and Instruments.}

All chemicals and reagents were commercially available and used as received without further purification. 3-(4,5-dimethyl-2-thiazolyl)-2,5-diphenyltetrazolium bromide (MTT) were purchased from Sigma-Aldrich and used as received. For cell culture, Dulbecco's Modified Eagle's Medium (DMEM), fetal bovine serum (FBS), penicillin, streptomycin solution, CellMask Green were purchased from Invitrogen. ${ }^{1} \mathrm{H}$ and ${ }^{13} \mathrm{C}$ NMR spectra were measured on a Bruker ARX 400 NMR spectrometer using $\mathrm{CDCl} 3$ and DMSO-d6 as solvents and tetramethylsilane (TMS; $\delta=0 \mathrm{ppm}$ ) was chosen as 
internal reference. High-resolution mass spectra (HR-MS) were obtained on a Finnigan MAT TSQ 7000 Mass Spectrometer System operated in a MALDI-TOF mode. Absorption spectra were measured on a Milton Roy Spectronic 3000 Array spectrophotometer. Steady-state photoluminescence (PL) spectra were measured on a Perkin-Elmer spectrofluorometer LS 55. Absolute fluorescence quantum yield was measured by a calibrated integrating sphere (Labsphere). Laser confocal scanning microscope images were collected on Zeiss laser scanning confocal microscope (LSM 800) and analyzed using ZEN 2009 software (Carl Zeiss). The statistical analysis of cells was measured by flow cytometry (BD FACS Aria IIIu). Fluorescence intensity that was used for quantative analysis was obtained on a microplate reader (Varioskan LUX multimode microplate reader) using black 96-well plates (Thermo Scientific, light-tight, flat bottom, nonsterile).

\section{Strain information}

S. aureus (ATCC 6538), B. subtilis (ATCC 6633), M. luteus (ATCC 9341), E. coli (8099), K. pneumoniae (ATCC 700603), S. marcescens (CMCC 41002), C. albicans (ATCC 10231), S. cerevisiae (ATCC 9763).

\section{Bacterial staining}

A single colony of bacteria on solid culture medium was transferred to $5 \mathrm{~mL}$ of liquid culture medium and grown at $37^{\circ} \mathrm{C}$ for $10 \mathrm{~h}$. After reaching logarithmic phase, $500 \mu \mathrm{L}$ of bacteria was transferred to a $1.5 \mathrm{~mL}$ microcentrifuge tube. Bacteria were harvested by centrifuging at $11700 \mathrm{~g}$ for $3 \mathrm{~min}$. Bacteria were harvested by centrifuging at 11700 $\mathrm{g}$ for $3 \mathrm{~min}$. After removal of supernatant, bacteria were either killed by $200 \mu \mathrm{L} 75 \%$ alcohol (dead bacteria) or dealt with equal amount of PBS (living bacteria), following by washing with PBS. Then, $1 \mathrm{~mL}$ dye solution in saline at appropriate concentration was added into the microcentrifuge tube. After dispersing with vortex, the bacteria were incubated in a shaking incubator at $37{ }^{\circ} \mathrm{C}$ for designed time. Finally imaged by ZEISS LSM800.

\section{Fungal staining}

A single colony of bacteria on solid culture medium was transferred to $5 \mathrm{~mL}$ of liquid culture medium and grown at $25^{\circ} \mathrm{C}$ for $10 \mathrm{~h}$. After reaching logarithmic phase, $500 \mu \mathrm{L}$ of bacteria/fungi was transferred to a $1.5 \mathrm{~mL}$ microcentrifuge tube. Fungi were 
harvested by centrifuging at $11700 \mathrm{~g}$ for 3 min. Fungi were harvested by centrifuging at $11700 \mathrm{~g}$ for $3 \mathrm{~min}$. After removal of supernatant, bacteria were either killed by 200 $\mu \mathrm{L} 75 \%$ alcohol (dead bacteria) or dealt with equal amount of PBS (living bacteria), following by washing with PBS. Then, $1 \mathrm{~mL}$ dye solution in saline at appropriate concentration was added into the microcentrifuge tube. After dispersing with vortex, the bacteria were incubated in a shaking incubator at $25^{\circ} \mathrm{C}$ for designed time. Finally imaged by ZEISS LSM800.

\section{Biofilm imaging}

An overnight culture of microbeswas subcultured at an OD600 of 0.5 into relevant medium (S. aureus: TSB supplemented with glucose. E. coli: LB supplemented with casein hydrolysate. C. albicans: YPD broth). Then, $2 \mathrm{~mL}$ of bacteria/fungi suspension was added to a confocal dish. The dishes were static cultured at $37{ }^{\circ} \mathrm{C}$. After $72 \mathrm{~h}$, the medium was removed and the plates were washed with sterile PBS. Then, $1 \mathrm{~mL}$ of the probes was added into the dishes incubated for designed time. Finally imaged by ZEISS LSM800.

\section{Quantitation of E. coli with different viabilities by plate reader}

A single colony of E. coli on solid culture medium was transferred to $5 \mathrm{~mL}$ of liquid culture medium and grown at $37^{\circ} \mathrm{C}$ for $10 \mathrm{~h}$. After reaching logarithmic phase, $500 \mu \mathrm{L}$ of culture suspension was transferred to a $1.5 \mathrm{~mL}$ microcentrifuge tube. Bacteria were harvested by centrifuging at $11700 \mathrm{~g}$ for $3 \mathrm{~min}$. After removal of supernatant, bacteria were either killed by $1.5 \mathrm{~mL} 75 \%$ alcohol (dead bacteria) or dealt with equal amount of PBS (living bacteria). Then live and dead bacteria were mixed at different ratios (L/D ratio), i.e., $0,10 \%, 30 \%, 50 \%, 70 \%, 90 \%$ and $100 \%$. Next, bacteria were harvested by centrifuging at $11700 \mathrm{~g}$ for $3 \mathrm{~min}$. Then, $200 \mu \mathrm{L}$ dye solution in buffer $(\mathrm{pH}=10)$ at appropriate concentration was added into the microcentrifuge tube. After dispersing with vortex, $100 \mu \mathrm{L}$ suspension were transferred into 96-well plate and incubated at $37{ }^{\circ} \mathrm{C}$ for designed time. Finally, the fluorescence peaks were recorded by microplate reader (Varioskan LUX multimode microplate reader) using black 96-well plates (Thermo Scientific, lighttight, flat bottom, non-sterile). The integrated intensities of the blue $(460 \pm 5 \mathrm{~nm})$ and red $(730 \pm 5 \mathrm{~nm})$ emission of suspensions excited at $310 \pm 10$ $\mathrm{nm}$ and $519 \pm 10 \mathrm{~nm}$ were acquired, and the red/blue fluorescence ratios (Ratio R/B ) 
were calculated for each proportion of live/dead E. coli. Each point represents the mean of four measurements.

\section{Quantitation of bacteria/fungi with different viabilities by flow cytometry.}

A single colony of $E$. coli on solid culture medium was transferred to $5 \mathrm{~mL}$ of liquid culture medium and grown at $37{ }^{\circ} \mathrm{C}$ for $10 \mathrm{~h}$. After reaching logarithmic phase, $500 \mu \mathrm{L}$ of bacteria suspension was transferred to a $1.5 \mathrm{~mL}$ microcentrifuge tube. Bacteria/fungi were harvested by centrifuging at $11700 \mathrm{~g}$ for $3 \mathrm{~min}$. After removal of supernatant, bacteria were either killed by $1.5 \mathrm{~mL} 75 \%$ alcohol (dead bacteria) or dealt with equal amount of PBS (living bacteria). Then live and dead bacteria/fungi were mixed at different ratios (L/D ratio), i.e., 0, $10 \%, 30 \%, 50 \%, 70 \%, 90 \%$ and $100 \%$. Next, bacteria/fungi were harvested by centrifuging at $11700 \mathrm{~g}$ for $3 \mathrm{~min}$. Then, $2 \mathrm{~mL}$ dye solution in buffer $(\mathrm{pH}=10)$ at appropriate concentration was added into the microcentrifuge tube. After dispersing with vortex, the suspensions were incubated at $37{ }^{\circ} \mathrm{C}$ for designed time. Finally, the bacteria were measured by flow cytometry (BD FACS Aria IIIu). The integrated intensities of the blue $(450 \pm 40 \mathrm{~nm})$ and red $(780 \pm$ $60 \mathrm{~nm}$ ) emission of suspensions excited at $405 \mathrm{~nm}$ and $561 \mathrm{~nm}$ were acquired, and the red/blue fluorescence ratios (Ratio R/B ) were calculated for each proportion of living/dead E. coli. Each point represents the mean of 5000 counts.

\section{Quantitation of bacteria/fungi with different viabilities by laser scanning confocal microscope}

A single colony of $E$. coli on solid culture medium was transferred to $5 \mathrm{~mL}$ of liquid culture medium and grown at $37^{\circ} \mathrm{C}$ for $10 \mathrm{~h}$. After reaching logarithmic phase, $500 \mu \mathrm{L}$ of bacteria was transferred to a $1.5 \mathrm{~mL}$ microcentrifuge tube. Bacteria were harvested by centrifuging at $11700 \mathrm{~g}$ for $3 \mathrm{~min}$. After removal of supernatant, bacteria were either killed by $1.5 \mathrm{~mL} 75 \%$ alcohol (dead bacteria) or dealt with equal amount of PBS (living bacteria). Then live and dead bacteria were mixed at different ratios (L/D ratio), i.e., 0, $10 \%, 30 \%, 50 \%, 70 \%, 90 \%$ and $100 \%$. Next, bacteria were harvested by centrifuging at $11700 \mathrm{~g}$ for $3 \mathrm{~min}$. Then, $100 \mu \mathrm{L}$ dye solution in buffer $(\mathrm{pH}=10)$ at appropriate concentration was added into the microcentrifuge tube. After dispersing with vortex, the suspensions were incubated at $37{ }^{\circ} \mathrm{C}$ for designed time. Finally, the confocal fluorescent images were obtained with Zeiss LSM 800 confocal laser scanning microscope. Different given percent live E. coli were stained with DCQA and TPE- 
2BA. Viability of E. coli suspension were calculated by CLSM images of each given percentage of live E. coli.

\section{Experimental procedures for minimum inhibition concentration (MIC) test}

A single colony of S. aureus, E. coli or C. albicans was grown in Mueller-Hinton broth. After reaching logarithmic phase, $1 \times 10^{6}$ cells $\mathrm{mL}^{-1}$ were adjusted for MIC test. DCQA and TPE-2BA were diluted in gradient through a sterile MH broth. After the probes had been diluted, a volume of the standardized inoculum equal to the volume of the diluted probes was added to each dilution vessel, bringing the microbial concentration to approximately $5 \times 10^{5}$ cells $\mathrm{mL}^{-1}$. The 96-well plates were incubated in incubator at $37^{\circ} \mathrm{C}$ for $24 \mathrm{~h}$. After incubation, the series of dilution vessels is observed for microbial growth, usually indicated by turbidity. The last tube in the dilution series that does not demonstrate was recorded as the MIC values of the probes.

\section{Author Contribution}

W. H., Z. Z., H. B. contributed equally in this work. W. H. and Z. Z. conceived the original idea for this study. W. H., Z.Z. and H. B. designed the experiments. W. H. and Z. Z. designed the molecules. Z. Z. synthesized the molecules and did photophysical experiments. W. H. did all biological experiments including bacterial culture, imaging, detection, characterization and calculation. B. Z. T. supervised the whole process. B. Z. T., W. H., Z. Z., H. B. and R. T. K. K. discussed for the manuscript. and prepared the manuscript. W. H. wrote the manuscript, and Z. Z., H. B. and R. T. K. K. revised the manuscript. All the authors participated in data analysis.

\section{Acknowledgment}

We greatly appreciate the financial support from the National Natural Science Foundation of China (21788102), the Research Grants Council of Hong Kong (C600917G, 16305618, N_HKUST609/19, and A-HKUST605/16), the Innovation and Technology Commission (ITC-CNERC14SC01), National Key Research and Development Program of China (2018YFE0190200), and the Technology Plan of Shenzhen (JCYJ20170818113602462 and JCYJ20180507183832744).

\section{Conflict of Interest}

The authors declare no conflict of interest. 


\section{Reference}

1. Stewart, E. J., Growing unculturable bacteria. J Bacteriol 2012, 194 (16), 4151 60.

2. Staley, J. T.; Konopka, A., Measurement of in situ activities of nonphotosynthetic microorganisms in aquatic and terrestrial habitats. Annual review of microbiology 1985, 39 (1), 321-346.

3. Browne, H. P.; Forster, S. C.; Anonye, B. O.; Kumar, N.; Neville, B. A.; Stares, M. D.; Goulding, D.; Lawley, T. D., Culturing of 'unculturable' human microbiota reveals novel taxa and extensive sporulation. Nature 2016, 533 (7604), 543546.

4. Barry, J. M., The great influenza: The story of the deadliest pandemic in history. Penguin UK: 2020.

5. Diamond, J. M.; Ordunio, D., Guns, germs, and steel. Books on Tape: 1999.

6. Breman, J. G.; Henderson, D. A., Diagnosis and management of smallpox. New England Journal of Medicine 2002, 346 (17), 1300-1308.

7. Pai, M.; Behr, M. A.; Dowdy, D.; Dheda, K.; Divangahi, M.; Boehme, C. C.; Ginsberg, A.; Swaminathan, S.; Spigelman, M.; Getahun, H.; Menzies, D.; Raviglione, M., Tuberculosis. Nat Rev Dis Primers 2016, 2, 16076.

8. Ashley, E. A.; Pyae Phyo, A.; Woodrow, C. J., Malaria. The Lancet 2018, 391 (10130), 1608-1621.

9. Perry, R. D.; Fetherston, J. D., Yersinia pestis--etiologic agent of plague. Clinical microbiology reviews 1997, 10 (1), 35-66.

10. Davey, H. M., Life, death, and in-between: meanings and methods in microbiology. Appl Environ Microbiol 2011, 77 (16), 5571-6.

11. Hartmann, M.; Berditsch, M.; Hawecker, J.; Ardakani, M. F.; Gerthsen, D.; Ulrich, A. S., Damage of the bacterial cell envelope by antimicrobial peptides gramicidin $\mathrm{S}$ and PGLa as revealed by transmission and scanning electron microscopy. Antimicrob Agents Chemother 2010, 54 (8), 3132-42.

12. Yuan, H.; Liu, Z.; Liu, L.; Lv, F.; Wang, Y.; Wang, S., Cationic conjugated polymers for discrimination of microbial pathogens. 2014, 26 (25), 4333-8.

13. Bai, H.; Chen, H.; Hu, R.; Li, M.; Lv, F.; Liu, L.; Wang, S., Supramolecular Conjugated Polymer Materials for in Situ Pathogen Detection. ACS Appl Mater Interfaces 2016, 8 (46), 31550-31557.

14. Coico, R., Gram staining. Current protocols in microbiology 2006, (1), A. 3C. 1-A. 3C. 2.

15. Le Blay, G.; Fliss, I.; Lacroix, C., Comparative detection of bacterial adhesion to Caco-2 cells with ELISA, radioactivity and plate count methods. J Microbiol Methods 2004, 59 (2), 211-21.

16. Cira, N. J.; Ho, J. Y.; Dueck, M. E.; Weibel, D. B., A self-loading microfluidic device for determining the minimum inhibitory concentration of antibiotics. Lab Chip 2012, 12 (6), 1052-9.

17. Feng, L.; Zhu, C.; Yuan, H.; Liu, L.; Lv, F.; Wang, S., Conjugated polymer nanoparticles: preparation, properties, functionalization and biological applications. Chem Soc Rev 2013, 42 (16), 6620-33.

18. He, X.; Xiong, L. H.; Zhao, Z.; Wang, Z.; Luo, L.; Lam, J. W. Y.; Kwok, R. T. K.; Tang, B. Z., AIE-based theranostic systems for detection and killing of pathogens. Theranostics 2019, 9 (11), 3223-3248.

19. Gao, M.; Tang, B. Z., Fluorescent Sensors Based on Aggregation-Induced Emission: Recent Advances and Perspectives. ACS Sens 2017, 2 (10), 1382-1399. 
20. Liu, H.; Xiong, L. H.; Kwok, R. T. K.; He, X.; Lam, J. W. Y.; Tang, B. Z., AIE Bioconjugates for Biomedical Applications. Advanced Optical Materials 2020, 8 (14).

21. Li, J.; Wang, J.; Li, H.; Song, N.; Wang, D.; Tang, B. Z., Supramolecular materials based on AIE luminogens (AIEgens): construction and applications. Chem Soc Rev 2020, 49 (4), 1144-1172.

22. Wang, Y.; Nie, J.; Fang, W.; Yang, L.; Hu, Q.; Wang, Z.; Sun, J. Z.; Tang, B. Z., Sugar-Based Aggregation-Induced Emission Luminogens: Design, Structures, and Applications. Chem Rev 2020, 120 (10), 4534-4577.

23. Gao, M.; Tang, B. Z., AIE-based cancer theranostics. Coordination Chemistry Reviews 2020, 402.

24. Mei, J.; Hong, Y.; Lam, J. W.; Qin, A.; Tang, Y.; Tang, B. Z., Aggregationinduced emission: the whole is more brilliant than the parts. Adv Mater 2014, 26 (31), 5429-79.

25. Vert, M.; Doi, Y.; Hellwich, K.-H.; Hess, M.; Hodge, P.; Kubisa, P.; Rinaudo, M.; Schué, F., Terminology for biorelated polymers and applications (IUPAC Recommendations 2012). Pure and Applied Chemistry 2012, 84 (2), 377-410.

26. Arciola, C. R.; Campoccia, D.; Montanaro, L., Implant infections: adhesion, biofilm formation and immune evasion. Nat Rev Microbiol 2018, 16 (7), 397-409.

27. Kowalski, C. H.; Morelli, K. A.; Schultz, D.; Nadell, C. D.; Cramer, R. A., Fungal biofilm architecture produces hypoxic microenvironments that drive antifungal resistance. Proc Natl Acad Sci U S A 2020, 117 (36), 22473-22483.

28. Flemming, H. C.; Wingender, J.; Szewzyk, U.; Steinberg, P.; Rice, S. A.; Kjelleberg, S., Biofilms: an emergent form of bacterial life. Nature reviews. Microbiology 2016, 14 (9), 563-75.

29. Koo, H.; Allan, R. N.; Howlin, R. P.; Stoodley, P.; Hall-Stoodley, L., Targeting microbial biofilms: current and prospective therapeutic strategies. Nat Rev Microbiol 2017, 15 (12), 740-755.

30. Boulos, L.; Prevost, M.; Barbeau, B.; Coallier, J.; Desjardins, R., LIVE/DEAD ${ }^{\circledR}$ BacLight ${ }^{\mathrm{TM}}$ : application of a new rapid staining method for direct enumeration of viable and total bacteria in drinking water. Journal of microbiological Methods 1999, 37 (1), 77-86.

31. Gupta, A.; Das, R.; Yesilbag Tonga, G.; Mizuhara, T.; Rotello, V. M., ChargeSwitchable Nanozymes for Bioorthogonal Imaging of Biofilm-Associated Infections. ACS Nano 2018, 12 (1), 89-94.

32. Ivleva, N. P.; Wagner, M.; Szkola, A.; Horn, H.; Niessner, R.; Haisch, C., Label-free in situ SERS imaging of biofilms. The journal of physical chemistry B 2010, 114 (31), 10184-10194.

33. Zhu, C.; Kwok, R. T. K.; Lam, J. W. Y.; Tang, B. Z., Aggregation-Induced Emission: A Trailblazing Journey to the Field of Biomedicine. ACS Applied Bio Materials 2018.

34. Alam, P.; He, W.; Leung, N. L. C.; Ma, C.; Kwok, R. T. K.; Lam, J. W. Y.; Sung, H. H. Y.; Williams, I. D.; Wong, K. S.; Tang, B. Z., Red AIE - Active Fluorescent Probes with Tunable Organelle-Specific Targeting. Advanced Functional Materials 2020, 30 (10).

35. Zheng, Z.; Liu, H.; Zhai, S.; Zhang, H.; Shan, G.; Kwok, R. T. K.; Ma, C.; Sung, H. H. Y.; Williams, I. D.; Lam, J. W. Y.; Wong, K. S.; Hu, X.; Tang, B. Z., Highly efficient singlet oxygen generation, two-photon photodynamic therapy and melanoma ablation by rationally designed mitochondria-specific near-infrared AIEgens. Chemical Science 2020, 11 (9), 2494-2503. 
36. Li, Y.; Zhao, Z.; Zhang, J.; Kwok, R. T.; Xie, S.; Tang, R.; Jia, Y.; Yang, J.; Wang, L.; Lam, J. W. J. A. F. M., A bifunctional aggregation-induced emission luminogen for monitoring and killing of multidrug-resistant bacteria. 2018, 28 (42), 1804632.

37. Roy, E.; Nagar, A.; Chaudhary, S.; Pal, S., AIEgen-Based Fluorescent Nanomaterials for Bacterial Detection and its Inhibition. ChemistrySelect 2020, 5 (2), $722-735$.

38. Liu, G. J.; Tian, S. N.; Li, C. Y.; Xing, G. W.; Zhou, L., Aggregation-InducedEmission Materials with Different Electric Charges as an Artificial Tongue: Design, Construction, and Assembly with Various Pathogenic Bacteria for Effective Bacterial Imaging and Discrimination. ACS Appl Mater Interfaces 2017, 9 (34), 28331-28338.

39. Sun, Y. Q.; Liu, J.; Zhang, H.; Huo, Y.; Lv, X.; Shi, Y.; Guo, W., A mitochondria-targetable fluorescent probe for dual-channel NO imaging assisted by intracellular cysteine and glutathione. J Am Chem Soc 2014, 136 (36), 12520-3.

40. King, S. M.; Claire, S.; Teixeira, R. I.; Dosumu, A. N.; Carrod, A. J.; Dehghani, H.; Hannon, M. J.; Ward, A. D.; Bicknell, R.; Botchway, S. W.; Hodges, N. J.; Pikramenou, Z., Iridium Nanoparticles for Multichannel Luminescence Lifetime Imaging, Mapping Localization in Live Cancer Cells. J Am Chem Soc 2018, 140 (32), 10242-10249.

41. Niu, G.; Zheng, X.; Zhao, Z.; Zhang, H.; Wang, J.; He, X.; Chen, Y.; Shi, X.; Ma, C.; Kwok, R. T. K.; Lam, J. W. Y.; Sung, H. H. Y.; Williams, I. D.; Wong, K. S.; Wang, P.; Tang, B. Z., Functionalized Acrylonitriles with Aggregation-Induced Emission: Structure Tuning by Simple Reaction-Condition Variation, Efficient Red Emission, and Two-Photon Bioimaging. J Am Chem Soc 2019, 141 (38), 15111-15120. 42. Wang, Y.; Chen, M.; Alifu, N.; Li, S.; Qin, W.; Qin, A.; Tang, B. Z.; Qian, J., Aggregation-Induced Emission Luminogen with Deep-Red Emission for ThroughSkull Three-Photon Fluorescence Imaging of Mouse. ACS Nano 2017, 11 (10), $10452-$ 10461.

43. Dong, N.-N.; Pedroni, M.; Piccinelli, F.; Conti, G.; Sbarbati, A.; RamírezHernández, J. E.; Maestro, L. M.; Iglesias-de la Cruz, M. C.; Sanz-Rodriguez, F.; Juarranz, A., NIR-to-NIR two-photon excited CaF2: Tm3+, Yb3+ nanoparticles: multifunctional nanoprobes for highly penetrating fluorescence bio-imaging. ACS Nano 2011, 5 (11), 8665-8671.

44. Radovic-Moreno, A. F.; Lu, T. K.; Puscasu, V. A.; Yoon, C. J.; Langer, R.; Farokhzad, O. C., Surface charge-switching polymeric nanoparticles for bacterial cell wall-targeted delivery of antibiotics. ACS nano 2012, 6 (5), 4279-4287.

45. Jennings, M. C.; Minbiole, K. P.; Wuest, W. M., Quaternary Ammonium Compounds: An Antimicrobial Mainstay and Platform for Innovation to Address Bacterial Resistance. ACS Infect Dis 2015, 1 (7), 288-303.

46. Zhao, E.; Hong, Y.; Chen, S.; Leung, C. W.; Chan, C. Y.; Kwok, R. T.; Lam, J. W.; Tang, B. Z., Highly fluorescent and photostable probe for long-term bacterial viability assay based on aggregation-induced emission. Adv Healthc Mater 2014, 3 (1), 88-96.

47. Wang, D.; Su, H.; Kwok, R. T. K.; Hu, X.; Zou, H.; Luo, Q.; Lee, M. M. S.; Xu, W.; Lam, J. W. Y.; Tang, B. Z., Rational design of a water-soluble NIR AIEgen, and its application in ultrafast wash-free cellular imaging and photodynamic cancer cell ablation. Chem Sci 2018, 9 (15), 3685-3693.

48. Chubukov, V.; Gerosa, L.; Kochanowski, K.; Sauer, U., Coordination of microbial metabolism. Nat Rev Microbiol 2014, 12 (5), 327-40. 
49. Eisenreich, W.; Dandekar, T.; Heesemann, J.; Goebel, W., Carbon metabolism of intracellular bacterial pathogens and possible links to virulence. Nat Rev Microbiol 2010, $8(6), 401-12$.

50. Cangelosi, G. A.; Meschke, J. S., Dead or alive: molecular assessment of microbial viability. Appl Environ Microbiol 2014, 80 (19), 5884-91.

51. Hu, D.; Deng, Y.; Jia, F.; Jin, Q.; Ji, J., Surface Charge Switchable Supramolecular Nanocarriers for Nitric Oxide Synergistic Photodynamic Eradication of Biofilms. ACS Nano 2020, 14 (1), 347-359.

52. Karatan, E.; Watnick, P., Signals, regulatory networks, and materials that build and break bacterial biofilms. Microbiol Mol Biol Rev 2009, 73 (2), 310-47.

53. Danese, P. N.; Pratt, L. A.; Kolter, R., Exopolysaccharide production is required for development of Escherichia coli K-12 biofilm architecture. Journal of bacteriology 2000, 182 (12), 3593-3596. 